\title{
Tätigkeitsbericht
}

\section{KRGÖ und FRQ in den Jahren 2018 und 2019}

Die Kommission für Rechtsgeschichte Österreichs (KRGÖ) der Österreichischen Akademie der Wissenschaften (ÖAW) und die Forschungsstelle für Rechtsquellenerschließung (FRQ) der Universität Wien konnten im Jahr 2018 gleich zwei Bände in der Reihe „Fontes Iuris“ herausbringen:

Der Band „Die Tiroler Landesordnungen von 1526, 1532 und 1573“ (= Fontes Iuris 26), herausgegeben von Josef PAUSER und KRGÖ-Mitglied Martin P. SCHENNACH, enthält zentrale Quellen zur Verfassungs-, Privatrechts- und Strafrechtsgeschichte Tirols in der Frühen Neuzeit, außer den im Buchtitel genannten auch noch die Tiroler Malefizordnung 1499 sowie die Tiroler Policeyordnung 1573.

Und im Rahmen des Langzeitprojekts „Die Wiener Stadtbücher 1395-1430" konnte der fünfte Teilband von Gerhard JARITZ und KRGÖ-Mitglied Christian NESCHWARA herausgegeben werden (= Fontes Iuris 10/5). Er enthält Eintragungen aus den Jahren 1418-1421, vor allem letztwillige Verfügungen („Geschäfte") und Verwandtschaftsweisungen, mit denen der Rechtsalltag im spätmittelalterlichen Wien rekonstruiert werden kann.

Große Fortschritte machte das an der KRGÖ angesiedelte und in Kooperation mit der FRQ sowie mit den rechtshistorischen Instituten der Universitäten Linz und Graz durchgeführte FWF-Projekt P 29774 „,Der Vertrag von Saint Germain“. Es wurden Vorträge gehalten, u.a. bei einer internationalen Konferenz in London im Juni 2019 und in der Gesamtsitzung der ÖAW vom 25. Oktober 2019, sowie einschlägige Aufsätze publiziert. Die Zeitschrift „zeitgeschichte“ widmete dem Vertrag von St. Germain einen Themenband, zu dem vier Projektmitarbeiter*innen Aufsätze beisteuerten und der von KRGÖ-Mitglied und FWF-Projektleiterin Anita ZIEGERHOFER herausgegeben wurde. Der große Kommentar zum Vertrag von St. Germain, der im Mittelpunkt des Projekts steht, soll 2020 erscheinen.

Vom 27.-29. September 2018 fand im Theatersaal der ÖAW die internationale Tagung „Der Vertrag vom Saint Germain im Kontext der europäischen Nachkriegsordnung“ statt. Veranstalter waren neben KRGÖ und FRQ auch das Institut für Neuzeit- und Zeitgeschichtsforschung der ÖAW sowie das FWF-Projekt „St. Germain“; die Veranstaltung wurde vom Beirat des Bundeskanzleramtes für das Gedenk- und Erinnerungsjahr 2018 finanziell unterstützt. Neun FWF-Projektmitarbeiter*innen sowie 16 weitere Wissenschafter*innen aus Bulgarien, Deutschland, Frankreich, Italien, Kanada, Polen und Ungarn sowie aus Österreich hielten Vorträge und vernetzten so das FWF-Projekt mit der internationalen Forschungslandschaft. Die Publikation der Tagungsbeiträge erfolgte in Band 2019/2 der BRGÖ, siehe dort zu weiteren Details.

Die Pariser Friedenskonferenzen, in deren Rahmen der Vertrag von St. Germain am 10. September 1919 unterzeichnet wurde, wurden immer wieder mit dem Wiener Kongress 1814/15 verglichen und kontrastiert. Ende 2019 erschien in den „Denkschriften der philosophisch-historischen Klasse" der ÖAW als Band 517 der zweiteilige Tagungsband „Der Wiener Kongress 1814/15“, herausgegeben von Thomas OLECHOWSKI, Brigitte MAZOHL, Karin SCHNEIDER, Reinhard STAUBER, Werner TELESKO, Elisabeth HILSCHER und Eva Maria WERNER. Er ging aus zwei Tagungen 
hervor, die 2015 an der ÖAW sowie an der Universität Innsbruck zum zweihundertjährigen Jubiläum des Kongresses stattgefunden hatten, und enthält nicht nur Beiträge zur Rechtsgeschichte und politischen Geschichte, sondern auch zur Kunst- und Musikgeschichte sowie zur Erinnerungskultur dieses Jahrhundertereignisses. Die 29 Autor*innen stammen aus Deutschland, Frankreich, Großbritannien, Italien, Norwegen, Polen, der Schweiz, Tschechien und den USA sowie aus Österreich.

Im Jahr 2018 jährte sich sowohl die Gründung der Ersten Republik Österreich als auch der Zweiten Polen zum hundertsten Mal. Am 27. Juni 2018 gedachte die FRQ dieser Ereignisse mit einer internationalen Tagung, die gemeinsam mit dem Hans Kelsen-Institut, dem Polnischen Institut Wien sowie dem Wissenschaftlichen Zentrum in Wien der Polnischen Akademie der Wissenschaften organisiert wurde. Unter dem Titel „Parlamente und Verfassungen in Polen und in Österreich 1918 bis 1921“ hielten neun Vortragende aus Polen und Österreich Referate im Senatssaal der Universität Wien; ausgewählte Beiträge dieser Veranstaltung werden 2020 im Band 41 der Schriftenreihe des Hans Kelsen-Instituts erscheinen.

Zum 90. Geburtstag des ehemaligen KRGÖ-Mitglieds w.M. Gerald STOURZH organisierte der Obmann der KRGÖ am 28. Mai 2019 einen Festakt im Theatersaal, bei dem, nach einleitenden Worten durch ÖAW-Präsident Anton ZeILINGER, k.M.I. Wolfgang MuELLER eine Laudatio und k.M.A. Winfried SCHULZE einen Festvortrag über „Die Geburt der Idee von Freiheit und Gleichheit aus der Ungleichheit" hielten.
Frau Mag. Laura Rosemarie RATHMANNER, die bis Juli 2018 als FWF-finanzierte Projektmitarbeiterin an der Universität Linz angestellt war, blieb noch weitere 13 Monate, von August 2018 bis August 2019, für das FWF-Projekt „St. Germain“ tätig, nunmehr jedoch als drittmittelfinanzierte Mitarbeiterin der KRGÖ selbst (die somit erstmals seit 2012 wieder über eine Mitarbeiterin verfügte).

Auch im Bereich der FRQ ergaben sich mehrere personelle Veränderungen: Der Dienstvertrag von Frau Univ.-Ass. Mag. Dr. Kamila StaudiglCieCHOWICZ LL.M. lief mit Jahresende 2018 aus und konnte aufgrund der Kettenvertragsregelung nicht weiter verlängert werden. Mit 15. März 2019 wurde Frau Mag. Carmen KLEINSZIG neue Universitätsassistentin im Rahmen der FRQ. Frau Katharina BERNOLD trat im August 2019 eine zehnmonatige Bildungskarenz an.

Im Jahr 2018 verbrauchte die FRQ $€ 74.376,94$ aus Eigenmitteln der Universität Wien, die KRGÖ $€ 5.190,64$ aus Eigenmitteln der ÖAW. Dazu kamen $€ 39.920,56$ von der Akademie der Wissenschaften zu Göttingen für das gemeinsame Projekt „Erschließung der Akten des Reichshofrates", $€ 18.194,71$ vom FWF und $€ 18.660,22$ von sonstigen Drittmittelgebern. Der gemeinsame Drittmittelanteil von FRQ und KRGÖ betrug somit $49,11 \%$.

Im Jahr 2019 verbrauchte die FRQ $€ 72.644,12$ aus Eigenmitteln der Universität Wien, die KRGÖ $€$ 8.833,60 aus Eigenmitteln der ÖAW. Dazu kamen $€ 27.910,03$ von der Akademie der Wissenschaften zu Göttingen und $€ 31.357,90$ vom FWF. Der gemeinsame Drittmittelanteil betrug somit $42,11 \%$. 